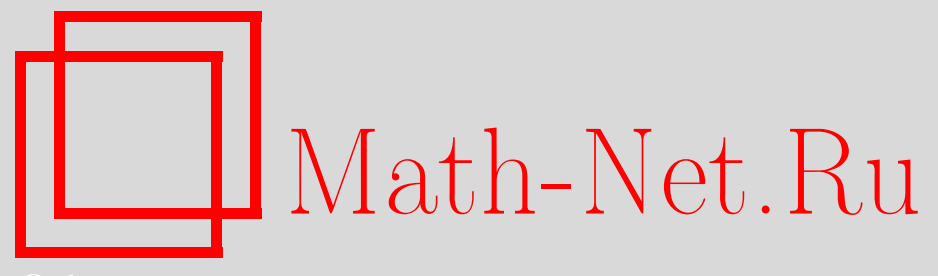

Э. Э. Гасанов, Ю. П. Луговская, Константный в худшем случае алгоритм поиска идентичных объектов, Дискрет. матем., 1999, том 11, выпуск 4, 139-144

DOI: https://doi.org/10.4213/dm396

Использование Общероссийского математического портала Math-Net.Ru подразумевает, что вы прочитали и согласны с пользовательским соглашением http://www.mathnet.ru/rus/agreement

Параметры загрузки:

IP : 54.198 .187 .58

26 апреля 2023 г., 14:01:01

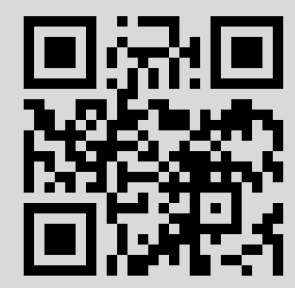




\title{
Константный в худшем случае алгоритм поиска идентичных объектов
}

\author{
(c) 1999 г. Э. Э. Гасанов, Ю. П. Луговская
}

В работе предлагается алгоритм поиска идентичных объектов, который при затратах памяти порядка $k^{2}$ почти всегда обеспечивает время поиска в множестве мощности $k$, равное в худшем случае шести элементарным операциям.

Работа выполнена при поддержке Российского фонда фундаментальных исследований, проект 98-01-00130.

\section{1. Введение}

В работе рассматривается задача поиска идентичных объектов в ее геометрической интерпретации, которая формулируется следующим образом. Дано конечное подмножество $V=\left\{y_{1}, \ldots, y_{k}\right\}$ точек из отрезка $[0,1]$ вещественной прямой. Требуется построить условный алгоритм, который для произвольной точки $x \in[0,1)$ (эта точка называется запросом) позволяет найти номер точки из множества $V$, которая совпадает с $x$ (если такая точка в $V$ существует), при условии, что мы умеем выполнять следующие операции над вещественными числами: арифметические операции (сложение, вычитание, умножение, деление, взятие целой части вещественного числа), операции сравнения и возможно некоторые другие простейшие операции. При этом допускается предобработка данных, которая может состоять в сортировке данных (множества $V$ ), а также в построении некоторых дополнительных структур. Известным алгоритмом решения этой задачи является алгоритм бинарного или дихотомического поиска (см., например, [1], с. 484-492). Здесь и далее мы будем пользоваться термином алгоритм, подразумевая условный алгоритм (или относительный алгоритм, см. [2], с. 44-45). Известно, что время поиска бинарного алгоритма в худшем случае и в среднем равно по порядку $\log _{2} k$, а требуемый объем памяти по порядку равен $k$. Здесь под временем поиска понимается количество выполненных элементарных операций, под объемом памяти количество ячеек для хранения вещественных чисел, куда можно поместить данные и дополнительные структуры, а худший случай и среднее берется по множеству всех возможных значений запроса, т.е. по множеству $[0,1)$. В [3] предлагается алгоритм с затратами памяти порядка $k$, в котором среднее время поиска равно константе, но время поиска в худшем случае имеет порядок $k$. В работах $[4,5]$ предложен алгоритм, который при объеме памяти порядка $k$ позволяет решать задачи в худшем случае за время порядка $\log _{2} k$, a в среднем за 2 шага. В данной работе предлагается алгоритм, который для почти всех задач поиска идентичных объектов (т.е. при вариации множества $V$ ) позволяет 
при объеме памяти порядка $k^{2}$ решать задачу в худшем случае за 6 элементарных операций.

\section{2. Описание алгоритма и основные результаты}

Опишем предлагаемый алгоритм. Пусть дано множество $V=\left\{y_{1}, y_{2}, \ldots, y_{k}\right\}$, в котором производится поиск. Это множество будем называть библиотекой. Выполним следующую предобработку. Упорядочим точки из $V$ в порядке возрастания и, чтобы не усложнять обозначения, далее считаем, что $y_{1}<y_{2}<\ldots<y_{k}$. Находим число

$$
d_{V}=\min _{2 \leqslant i \leqslant k}\left(y_{i}-y_{i-1}\right) \text {. }
$$

Пусть $m=] 1 / d_{V}\left[-\right.$ наименьшее целое, не меньшее, чем $1 / d_{V}$. Выделим место под массив целых длины $m$ и элементы массива обозначим $n_{i}, i=0,1,2, \ldots, m-1$. Разделим отрезок $[0,1]$ на $m$ равных частей:

$$
A_{i}=[i / m,(i+1) / m), \quad i=0,1, \ldots, m-2, \quad A_{m-1}=[(m-1) / m, 1] .
$$

В каждую часть может попасть не более одной точки из множества $V$. Теперь заполним массив $n_{i}$ следуюуцим образом:

$$
n_{i}= \begin{cases}-1, & \text { если в } A_{i} \text { нет точки из } V, \\ q & \text { в противном случае. }\end{cases}
$$

где $q$ - номер точки из $V$, которая попала в $A_{i}, i=0,1,2, \ldots, m-1$.

После того как сделана данная предобработка, поиск будем осуществлять следующим образом. Пусть нам дан запрос $x \in[0,1)$. Вычислим $j=[x m]$, целую часть числа $x \mathrm{~m}$. Понятно, что $x \in A_{j}$. Если $n_{j}$ равно -1 , то в библиотеке $V$ нет числа равного $x$. В противном случае сравниваем $y_{n_{j}}$ с $x$ и если они равны, то номер $n_{j}$ является ответом задачи, иначе ответ пуст. Тем самым в худшем случае мы выполняем одну операцию умножения, одну операцию взятия целой части, одну операцию сравнения целых чисел, одну операцию сравнения вещественных чисел и две операции извлечения элемента массива, всего 6 операций. Объем памяти, необходимый данному алгоритму, равен сумме объемов массивов целых чисел длины $m$ и вещественных чисел длины $k$. Ниже приводятся результаты, оценивающие величину $m$.

Пусть $k$ - натуральное число, большее 1 , и $\xi_{1}, \xi_{2}, \ldots, \xi_{k}$ - независимые равномерно распределенные на отрезке $[0,1]$ случайные величины. Пусть

$$
d\left(\xi_{1}, \ldots, \xi_{k}\right)=\min _{1 \leqslant i<j \leqslant k}\left|\xi_{i}-\xi_{j}\right|
$$

Эта величина исследовалась, например, в $[6,7]$.

Пусть $r$ - вещественное число и $f(k, r)=\mathbf{P}\left(d\left(\xi_{1}, \ldots, \xi_{k}\right) \geqslant r\right)$ - вероятность того, что минимальное расстояние между парами различных точек $\xi_{i}, i=1,2, \ldots, n$, не меньше $r$.

Если считать, что библиотеки $V$ получаются случайным независимым бросанием $k$ точек на отрезок $[0,1]$, где вероятность попадания в любые два отрезка из $[0,1]$ одинаковой длины одинакова, то $f(k, r)$ равна доле множества $k$-элементных библиотек, у которых минимальное расстояние между любыми двумя точками не меньше, чем $r$, по отношению к множеству всех библиотек мощности $k$.

Справедлива следующая теорема. 
Теорема 1. Прu $0 \leqslant r \leqslant 1 /(k-1)$

$$
f(k, r)=(1-(k-1) r)^{k}
$$

$u f(k, r)=0$ npu $r<0 u r>1 /(k-1)$.

Чтобы привести следствие из этой теоремы, описывающее асимптотическое поведение $f(k, r)$, введем обозначения, обычно принятые при описании асимптотических оценок.

Будем писать $\alpha(n)=o(1)$, если $\lim _{n \rightarrow \infty} \alpha(n)=0$.

Будем писать $A(n)=o(B(n))$, если $A(n)=B(n) o(1)$.

Скажем, что $A(n)$ асимптотически не превосходит $B(n)$ при $n \rightarrow \infty$ и будем писать в этом случае $A \lesssim B$, если существует $\alpha(n)=o(1)$ такое, что начиная с некоторого номера $n_{0}$,

$$
A(n) \leqslant(1+\alpha(n)) B(n) .
$$

Если $A \lesssim B$ и $B \lesssim A$, то будем говорить, что $A$ и $B$ асимптотически равны при $n \rightarrow \infty$ и будем писать в этом случае $A \sim B$.

Будем писать $A<B$, если существует такая положительная константа $c$, что $A(n) \leqslant c B(n)$, начиная с некоторого номера $n_{0}$.

Если $A<B$ и $B \leq A$, то будем говорить, что $A$ и $B$ равны по порядку при $n \rightarrow \infty$.

Теорема 2. Пусть $r_{k}-$ последователъность вещественных чисел такал, что $0 \leqslant r_{k} \leqslant 1 /(k-1)$. Тогдa

$$
f\left(k, r_{k}\right)= \begin{cases}0, & \text { если } 1 / r_{k}=o\left(k^{2}\right) \\ e^{-1 / c}, & \text { если } 1 / r_{k} \sim c k^{2}, c-\text { постояннал } \\ 1, & \text { если } k^{2}=o\left(1 / r_{k}\right) .\end{cases}
$$

Отсюда следует, что если в нашем распоряжении имеется объем памяти размеpa $c k^{2}$, то доля библиотек мощности $k$, для которых описанным алгоритмом мы можем находить ответ за 6 элементарных операций, равна $e^{-1 / c}$. А если в нашем распоряжении имеется объем памяти больший по порядку, чем $k^{2}$, то для почти всех библиотек мы можем находить ответ за 6 шагов. С другой стороны, если у нас имеется объем памяти, меньший по порядку, чем $k^{2}$, то почти всегда мы не сможем воспользоваться описанным выше алгоритмом поиска.

Обозначим через $\bar{d}(k)$ среднее значение величины $d\left(\xi_{1}, \ldots, \xi_{k}\right)$.

Теорема 3. Справедливо равенство

$$
\bar{d}(k)=1 /\left(k^{2}-1\right) .
$$

Отсюда следует, что в среднем достаточно иметь $k^{2}$ памяти, чтобы обеспечить время поиска в 6 операций.

\section{3. Доказательство основных результатов}

Доказательство теоремы 1 . Пусть $l$ - вещественное число из отрезка $[0,1], r-$ вещественное число, $k$ - целое число, большее $1, n-$ такое целое число, что 
$1 \leqslant n \leqslant k$. Пусть $\xi_{1}, \xi_{2}, \ldots, \xi_{k}-$ независимые равномерно распределенные на отрезке $[0,1]$ случайные величины. Обозначим через $B(n, r, l)$ событие, состоящее в том, что точки $\xi_{1}, \xi_{2}, \ldots, \xi_{n}$ попадают в отрезок $[0, l]$, и минимальное расстояние между парами различных точек $\xi_{i}, i=1,2, \ldots, n$, не меньше $r$. Пусть $f(n, r, l)=\mathbf{P}(B(n, r, l))$. Понятно, что если $r<0$, то $f(n, r, l)=l^{n}$, а при $r>l /(n-1), f(n, r, l)=0$. Поэтому мы будем рассматривать только случай, когда $0 \leqslant r \leqslant l /(n-1)$.

Лемма 1. Если $0 \leqslant r \leqslant l /(n-1)$, mo $f(n, r, l)=(l-(n-1) r)^{n}$.

Доказательство проведем индукцией по $n$.

В качестве базиса индукции рассмотрим случай $n=2$.

Поскольку возможны два равновероятных события, когда $\xi_{1}<\xi_{2}$, и когда $\xi_{2}<\xi_{1}$, то достаточно рассмотреть первую ситуацию и удвоить полученный результат. Поскольку в этом случае $\xi_{1}$ может меняться от 0 до $l-r$, а $\xi_{2}-$ от $x_{1}+r$ до $l$,

$$
f(2, r, l)=2 \int_{0}^{l-r} d \xi_{1} \int_{\xi_{1}+r}^{l} d \xi_{2}=2 \int_{0}^{l-r}\left(l-x_{1}-r\right) d \xi_{1}=(l-r)^{2} .
$$

Рассмотрим индуктивный переход. Пусть утверждение леммы справедливо для любого натурального $q<n$ и любого вещественного $l \in[0,1]$.

Через $A_{i}$ обозначим событие, состоящее в том, что случайная величина $\xi_{i}$ максимальна среди $\xi_{1}, \ldots, \xi_{n}$, здесь $i=1, \ldots, n$. Понятно, что если $i, j \in\{1, \ldots, n\}$ и $i \neq j$, то $A_{i} \cap A_{j}=\varnothing$, кроме того $\mathbf{P}\left(A_{i}\right)=1 / n$ для любого $i \in\{1, \ldots, n\}$. Легко видеть, что

$$
\mathbf{P}(B(n, r, l))=\sum_{i=0}^{n} \mathbf{P}\left(A_{i} \cap B(n, r, l)\right)=n \mathbf{P}\left(A_{n} \cap B(n, r, l)\right) .
$$

Поскольку в случае события $A_{n} \cap B(n, r, l)$ величина $\xi_{n}$ может меняться от $(n-1) r$ до $l$, а остальные $n-1$ величины располагаются на отрезке $\left[0, x_{n}-r\right]$ и должны находиться на расстоянии не менее $r$, то согласно предположению индукции

$$
\begin{aligned}
f(n, r, l) & =\mathbf{P}(B(n, r, l))=n \mathbf{P}\left(A_{1} \cap B(n, r, l)\right) \\
& =n \int_{(n-1) r}^{l} \mathbf{P}\left(B\left(n-1, r, x_{n}-r\right)\right) d \xi_{n} \\
& =n \int_{(n-1) r}^{l}\left(x_{n}-r-(n-2) r\right)^{n-1} d \xi_{n}=(l-(n-1) r)^{n} .
\end{aligned}
$$

Тем самым лемма доказана.

Чтобы убедиться в справедливости утверждения теоремы 1 достаточно заметить, что $f(k, r)=f(k, r, 1)$.

Доказательство теоремы 2. Пусть $\alpha_{k}=o(k)$ при $k \rightarrow \infty$. Ясно, что

$$
\begin{aligned}
\lim _{k \rightarrow \infty}\left(1-\frac{\alpha_{k}}{k}\right)^{k} & =\lim _{k \rightarrow \infty}\left(\frac{k}{k-\alpha_{k}}\right)^{-k}=\lim _{k \rightarrow \infty}\left(\left(1+\frac{\alpha_{k}}{k-\alpha_{k}}\right)^{\left(k-\alpha_{k}\right) / \alpha_{k}}\right)^{-k \alpha_{k} /\left(k-\alpha_{k}\right)} \\
& =\lim _{k \rightarrow \infty} e^{-k \alpha_{k} /\left(k-\alpha_{k}\right)}=\lim _{k \rightarrow \infty} e^{-\alpha_{k}}
\end{aligned}
$$


Рассмотрим случай, когда $1 / r_{k}=o\left(k^{2}\right)$ при $k \rightarrow \infty$.

Это означает, что для некоторой последовательности $\alpha_{k} \rightarrow \infty$ при $k \rightarrow \infty$ выполняется равенство $r_{k}=\alpha_{k} / k^{2}$. Поскольку $r_{k} \leqslant 1 /(k-1)$, достаточно рассмотреть два подслучая: $\alpha_{k} \sim c k$, где $c-$ константа, не превышающая 1, и $\alpha_{k}=o(k)$. В первом подслучае

$$
f\left(k, r_{k}\right)=\left(1-\frac{(k-1) \alpha_{k}}{k^{2}}\right)^{k} \sim\left(1-\frac{c(k-1) k}{k^{2}}\right)^{k}=o(1)
$$

Так как во втором подслучае $(k-1) \alpha_{k} / k=o(k)$, согласно (1)

$$
\lim _{k \rightarrow \infty} f\left(k, r_{k}\right)=\lim _{k \rightarrow \infty}\left(1-\frac{(k-1) \alpha_{k}}{k^{2}}\right)^{k}=\lim _{k \rightarrow \infty} e^{-(k-1) \alpha_{k} / k}=\lim _{k \rightarrow \infty} e^{-\alpha_{k}}=0
$$

Рассмотрим случай, когда $1 / r_{k} \sim c k^{2}$ при $k \rightarrow \infty$, где $c-$ постоянная.

Поскольку $(k-1) / c k=o(k)$, согласно (1)

$$
\lim _{k \rightarrow \infty} f\left(k, r_{k}\right)=\lim _{k \rightarrow \infty}\left(1-\frac{k-1}{c k^{2}}\right)^{k}=\lim _{k \rightarrow \infty} e^{-(k-1) /(c k)}=e^{-1 / c}
$$

И наконец, рассмотрим случай, когда $k^{2}=o\left(1 / r_{k}\right)$ при $k \rightarrow \infty$.

Это означает, что для некоторой последовательности $\alpha_{k} \rightarrow 0$ при $k \rightarrow \infty$ выполняется равенство $r_{k}=\alpha_{k} / k^{2}$. Поскольку $(k-1) \alpha_{k} / k=o(k)$, согласно (1)

$$
\lim _{k \rightarrow \infty} f\left(k, r_{k}\right)=\lim _{k \rightarrow \infty}\left(1-\frac{(k-1) \alpha_{k}}{k^{2}}\right)^{k}=\lim _{k \rightarrow \infty} e^{-(k-1) \alpha_{k} / k}=\lim _{k \rightarrow \infty} e^{-\alpha_{k}}=1 \text {. }
$$

Тем самым теорема 2 доказана.

Доказателъство теоремъ 3. Обозначим через $F(x)$ функцию распределения случайной величины $d\left(\xi_{1}, \ldots, \xi_{k}\right)$.

$$
F(x)=\mathbf{P}\left(d\left(\xi_{1}, \ldots, \xi_{k}\right)<x\right)=1-\mathbf{P}\left(d\left(\xi_{1}, \ldots, \xi_{k}\right) \geqslant x\right)=1-f(k, x) .
$$

Тогда так как при $x \leqslant 0 F(x)=0$, а при $x \geqslant 1 /(k-1) F(x)=1$, используя формулу интегрирования по частям, нетрудно получить, что

$$
\begin{aligned}
\bar{d}(k) & =\int_{-\infty}^{\infty} x d F(x)=\int_{0}^{1 /(k-1)} x d F(x)=\left.x F(x)\right|_{0} ^{\frac{1}{k-1}}-\int_{0}^{1 /(k-1)} F(x) d x \\
& =\int_{0}^{1 /(k-1)} f(k, x) d x=\int_{0}^{1 /(k-1)}(1-(k-1) x)^{k} d x=\frac{1}{k^{2}-1} .
\end{aligned}
$$

Тем самым теорема 3 доказана.

Авторы выражают благодарность рецензенту за ценные замечания. 


\section{Список литературы}

1. Кнут Д., Искусство программирования для ЭВМ. Сортировка и поиск, т. 3. Мир, Москва, 1978.

2. Мальцев А.И., Алгоритмъ и рехурсивные функции. Наука, Москва, 1986.

3. Ершов А.П., О программировании арифметических операторов. Докл. АН CCCP (1958) 118, 427-430.

4. Гасанов Э.Э., Мгновенно решаемые задачи поиска. Дискретная математика (1996) 8, №3, 119-134.

5. Гасанов Э.Э., Фунхционалъно-сетевые базы данных и сверхбыстрые алгоритмы поиска. Изд. центр РГГУ, Москва, 1997.

6. Devroye L. Upper and lower class sequences for minimal uniform spacings. Zeitschrift fur Wahrscheinlichkeitstheorie und verwande Gebiete (1982) 61, № 2, 237-254.

7. Дейвид Г., Порядковъе статистики. Наука, Москва, 1979.

Статья поступила 16.10.1998.

Переработанный вариант поступил 22.07.1999. 